\title{
Practical considerations in the study of main-chain thermotropic liquid-crystalline polymers by vibrational microscopy
}

\author{
G. Ellis*, M. Gómez and C. Marco \\ Departamento de Física e Ingeniería, Instituto de Ciencia y Tecnología de Polímeros, CSIC, Juan de la Cierva 3, 28006-Madrid, Spain
}

Current approaches to the study of the mesomorphic properties of main-chain thermotropic liquid crystalline polymers by vibrational microscopy are discussed, highlighting with some examples the information available, emphasising advantages and disadvantages of the techniques, and offering perspectives on how new technological developments may affect the field.

\section{Introduction}

Over the last twenty years there has been a significant research effort, both in academic and industrial laboratories, on main-chain thermotropic liquid-crystalline polymers (TLCPs) containing rigid aromatic mesogenic units, undoubtedly due to the important technological implications derived from their self-ordering properties. In this respect, an enormous variety of liquid crystalline polymer structures with the mesogenic units forming part of the polymer backbone have been reported. Many of these materials can be considered experimental and have given rise to a large body of literature on ordered and disordered states in polymers. Others have developed into high performance advanced engineering materials, such as Vectra ${ }^{\mathrm{TM}}$ or $\mathrm{Xydar}^{\mathrm{TM}}$, etc., with a wide range of applications in the electronics, transport, aviation and aerospace industries.

The vast majority of TLCPs of this type exhibit either smectic or nematic mesophases [1], normally identified by polarised thermo-optical microscopy (TOA). In some TLCPs, the ordered liquid crystal structure can be "frozenin" allowing the examination of the morphology of the mesophase in the solid state [2]. The type and degree of anisotropy obtained can be complex and, unlike the case for monomeric liquid crystals, the incidence of amorphous and crystalline material can further complicate matters. The analysis of the degree of liquid crystalline order is generally approached by X-ray diffraction methods [1], and in some cases, for example in smectic mesophases, oriented samples must be studied in order to identify the type of mesophase.

There are a number of macroscopic studies reported in the literature, dating back to the early 1980 's, where vibrational spectroscopy, principally FT-IR, has been used for the characterisation of TLCPs. These can be essentially divided into two groups: orientational order [3-11], and thermotropic behaviour [5,12-26]. With respect to the thermotropic properties, both temperature variable IR [6,13,15,18,22-26] and Raman [19,24] spectroscopy have been employed, and clear correlations with the transition temperatures observed by TOA and DSC have been reported.

The application of vibrational microspectroscopy to this field is fairly recent, and only a small number of studies have appeared in the literature to date [27-31], exclusively 
dedicated to the study of orientation in processed materials. Thus, it is the purpose of this manuscript to describe current approaches to the study of the thermotropic properties of TLCPs by vibrational microscopy, highlighting with some examples the information available, emphasising the advantages and disadvantages of the techniques, and offering perspectives on how new technological developments may affect the field.

\section{Experimental details}

IR and Raman spectra were measured on the following instruments under different conditions.

1. Perkin Elmer System 2000 spectrometer incorporating a PE i-Series IR-microscope with a liquid nitrogen cooled MCT detector and IMAGE software. IR spectra were recorded from microscopic samples prepared between pressurised $\mathrm{KBr}$ windows in a CIC Photonics High Temperature Cell. The samples were heated to the isotropic phase and cooled to $30{ }^{\circ} \mathrm{C}$ at $10^{\circ} \mathrm{C} / \mathrm{min}$ 200 scans $(10 \mathrm{~min})$. Spectra were recorded at specific temperatures, or as a function of temperature at intervals of $10^{\circ} \mathrm{C}$ with 5 minutes settling time at each step. All spectra were recorded at a resolution of $4 \mathrm{~cm}^{-1}$. Aperture settings are given in the figures.

2. Perkin Elmer System 2000 spectrometer, fitted with a 2000R FT-Raman accessory. $1064 \mu \mathrm{m} \mathrm{Nd}^{3+}$ :YAG laser excitation, and a room temperature InGaAs detector. Spectra were recorded at a resolution of $4 \mathrm{~cm}^{-1}$ accumulating 50 scans $(10 \mathrm{~min})$, with a laser power at the sample of $200 \mathrm{~mW}$, and corrected for the instrument optical response function.

3. Jobin-Yvon T64000 with microscope. 100× objective, $4 \mathrm{~cm}^{-1}$ resolution, 1-2 $\mathrm{mW} \mathrm{Ar}^{+}$laser excitation $(514.5 \mathrm{~nm})$ at the sample, CCD detector. Courtesy of Dr. Tariq Jawhari.

4. Bruker RFS100/S with Ramanscope. 100× objective, $4 \mathrm{~cm}^{-1}$ resolution, $1,064 \mu \mathrm{m} \mathrm{Nd}^{3+}$ :YAG laser excitation, and a liquid nitrogen cooled Ge detector. Spectrum uncorrected. Courtesy of Dr. Santiago Sánchez.

Spectral data were processed using PE Spectrum and GRAMS software. The instrument used is indicated in each figure.

\section{Practical considerations}

Vibrational microscopy is, in essence, a group of hyphenated techniques where a microscope is the common denominator. Although it can be considered in many laboratories as a routine analytical method, the combination of the advantages and disadvantages of microscopic analysis with those of each spectroscopic method give rise to a series of conditioning factors which can have important repercussions depending on the type of material under study. The study of polymeric materials by vibrational microscopy is well documented in the literature [32-38], however, it is convenient to highlight some of the more important features with respect to TLCPs.

In a similar way to that observed in thermotropic molecular liquid crystals, TLCPs demonstrate mesomorphism (different liquid crystalline phases) and in many cases polymorphism (different crystalline forms) as a function of temperature and/or thermal history. However, unlike molecular liquid crystals, TLCPs are not homogeneous in phase structure, and tend to exhibit a phase distribution which is a function of the chemical nature of the material, macromolecular parameters such as the molecular weight, thermomechanical conditions imposed during sample preparation, and thermal parameters related to the mesophase temperature, the residence time, and the thermal history, especially in cases where memory effects are observed in the anisotropic phase, or the liquid crystalline phase structure can be "frozen-in" employing very high undercoolings by rapid quenching in cryogenic liquids. As a consequence, the study of LCPs is much more complicated than it first appears, and requires a combination of thermal and optical methods in order to carefully control the nature of the materials under study.

\section{Sample preparation/manipulation}

In principle, neither Raman microscopy nor IR microscopy, when reflection techniques using ATR or grazing angle objectives are used, present sampling difficulties. Both approaches are feasible when one is studying orientational parameters in injection moulded parts, extrusion oriented sheets or even fibres. However, for the study of thermotropic behaviour at a structural level, careful control of the thermomechanical parameters of the sample is required, particularly in the case of transmission IR microscopy, where the sample thickness must be adequate for both the thermooptical and IR studies.

A thin film is usually prepared from the isotropic state between two IR-transparent plates (typically $\mathrm{KBr}$ or $\mathrm{NaCl}$ ), and cooled to room temperature. These can then be conveniently placed in a temperature controlled cell. It should be pointed out that some LCPs are sensitive to the optical surface, and may undergo induced ordering as a result of surface defects or the nature of the material used. Further, orientation induced during preparation may be difficult to remove due to the highly viscous nature of the anisotropic melts, thus the necessity to hold the sample in the isotropic state to remove any residual order. In some materials, the onset of thermal decomposition coincides or is close to the isotropisation temperature, which make this method of preparation less viable. Another approach is to prepare the samples as a dispersion in $\mathrm{KBr}$. This allows the study of the sample from its original state as a function of temperature, without previously altering the thermal history. However, the disadvantages are that the high pressure used in the 
preparation of the disk may induce structural changes such as polymorphism, and the dilution of the LCP material in $\mathrm{KBr}$ prevents the formation of large area mesomorphic domains, and the observation of LCP textures by polarised light microscopy. The use of high pressure diamond windows may also modify the thermotropic behaviour of the system.

In principle, the temperature at which these materials are studied should not be a limiting factor. The isotropisation temperature tends to fall between $250-500{ }^{\circ} \mathrm{C}$ depending on the chemical structure of the TLCP. It is well known that all-aromatic TLCPs have very high transition temperatures which are dependent on the size and structure of the mesogenic units [39], and the introduction of flexible spacer units between the mesogenic units or substituents on the mesogen or in the spacer have the effect of reducing the transition temperatures, for example [40-43]. These temperatures pose no problem for the majority of the high-temperature arrangements available, the limiting factor being simply the proximity of the hottest surface of the cell to the sealant and/or adhesive in the microscope objective. As a consequence, cell design is paramount, for example a limit of around $200{ }^{\circ} \mathrm{C}$ can be encountered for a simple uncooled hot-cell in an FTIR microscope with open Cassegrain optics, whereas spectra have been recorded at temperatures in excess of $1000{ }^{\circ} \mathrm{C}$ in a confocal Raman microscope with a hot-cell employing a water-cooled external case [44].

In Raman microscopy, a further complication arises. The majority of these aromatic-based materials are highly fluorescent, making it difficult to obtain good quality spectra with conventional laser excitation frequencies, as can be seen in the example given in figure 1 . Thus, the selection of longer excitation wavelengths, or the use of NIR FT-Raman microscopy is necessary. In this case, the proximity of the Raman shifted radiation to the spectral range corresponding to blackbody emission generates a temperature limit of around $200{ }^{\circ} \mathrm{C}$ for the $\mathrm{Nd}^{3+}: \mathrm{YAG}$ exciting line at $1.064 \mu \mathrm{m}$, before the broad continuum becomes too intense to be able obtain a reasonable spectrum. Using a pulsed $[45,46]$ or chopped [47] laser source, with the corresponding modifications to the data collection electronics, removes this

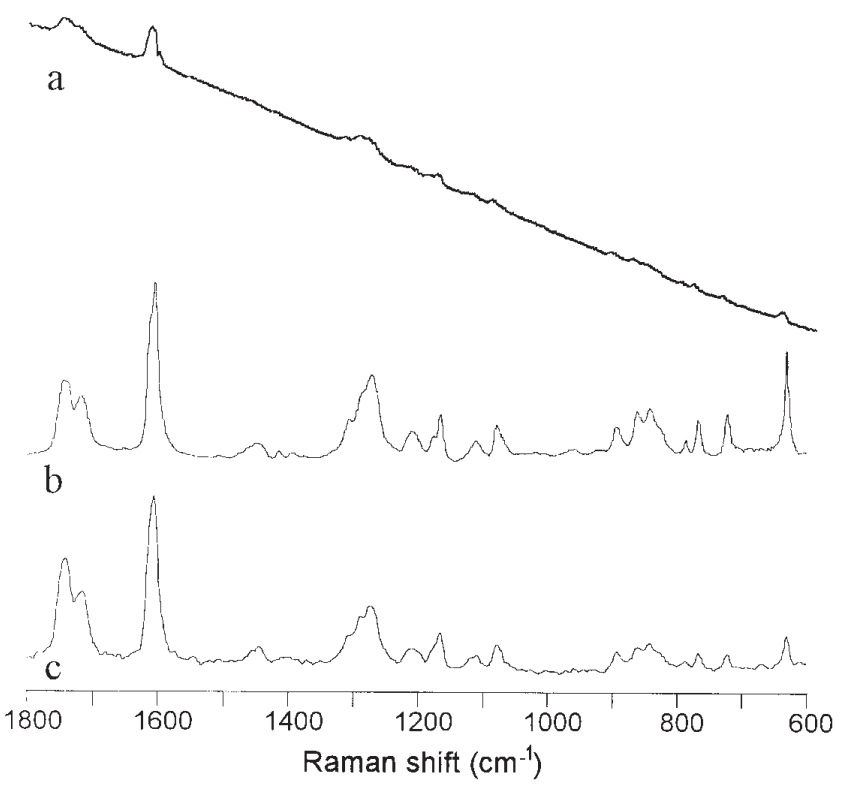

Figure 1. Raman spectra of a liquid crystal polyester, P7TOB (see later). Instruments used are a) 3, b) 2 and c) 4 as described in the experimental details.

impediment. However, to date instrument manufacturers do not provide these options as standard, suggesting as a solution the use of a simple waveband filter in the optical train, which effectively raises the high temperature limit to around $350{ }^{\circ} \mathrm{C}$, but at the expense of the Raman shifted region above the filter cut-off, commonly at around $2000 \mathrm{~cm}^{-1}$.

\section{Domain size and distribution}

Table I shows some typical theoretical spatial resolution limits for vibrational microscopy over a range of working wavelengths. The actual values vary from instrument to instrument, and depend on the microscope optics, in particular the numerical aperture of the objective used for collecting the spectrum. It is important to calculate these values before

Table I. Typical values for the theoretical limit ${ }^{1}$ of spatial resolution

\begin{tabular}{lcc}
\hline Technique & Spectral range $\left(\mathrm{cm}^{-1}\right)$ & Approximate resolution limits, $\Delta /(\mu \mathrm{m})$ \\
\hline $\mathrm{IR}^{2}$ & $4000-400$ & $3.7-37$ \\
Raman $^{3}$ & $3600-0$ & \\
& at $\lambda_{0}=0.5145 \mu \mathrm{m}$ & $1.4-1.1$ \\
& at $\lambda_{0}=0.633 \mu \mathrm{m}$ & $1.8-1.4$ \\
& at $\lambda_{0}=1.064 \mu \mathrm{m}$ & $3.8-2.3$ \\
\hline
\end{tabular}

1. The Rayleigh diffraction limit, $\Delta l=1,22 \mathrm{f} \lambda / \mathrm{d}$, where $\mathrm{f}=$ focal length of microscope objective, $\lambda=$ wavelength of radiation, and $\mathrm{d}=$ diameter of objective

2. For an objective with numerical aperture, $N A=0.6$

3. For an objective with $\mathrm{NA}=0.9$ 
embarking upon any experiment. In the study of mesophase distribution, it is convenient that the minimum domain size should fall above the spatial resolution limit defined by the spectroscopic technique. However, even though this is not the case, it is still possible to carry out mapping experiments similar to those used for the determination of compositional or structural heterogeneity polymer blends [37,48-50], generating a structural representation over specified areas of the sample as a function of the aperture used. Although structural details smaller than the diffraction limit can be observed in many birefringent textures, by careful sample preparation it is sometimes possible to generate regions of the sample with highly homogeneous mesomorphic structures, which can permit their study by vibrational microscopy.

Several recent developments in vibrational microscopy have improved the spatial resolution, and should be available in the near future. With FTIR microscopy using a synchrotron radiation source spectra have been recorded from polymeric materials at spatial resolutions below the diffraction limit [38,51]. Perhaps the most spectacular advances have been achieved in coupling vibrational spectroscopy to near-field optical microscopy (NFOM) experiments. Using both Raman scanning near-field optical microscopy (RSNOM) [52,53] and IR-NFOM [54] one can obtain spectra with sub-micrometre spatial resolution, and up to $17 \mathrm{~nm}$ resolution has been reported for mid-IR imaging experiments in simultaneous measurements with atomic force microscopy [55].

\section{Thermotropic behaviour}

Some general comments can be made with regard to the sensitivity of vibrational microscopy to the thermotropic behaviour of TLCPs. Vibrational spectroscopy is generally highly sensitive to crystallinity and polymorphism in polymers, and both the estimation of crystallinity and the identification of different crystalline forms has been shown to be feasible with macroscopic measurements [19,24,42]. Figure 2 shows the FT-IR and FT-Raman microspectra of poly(oxyheptamethylene-oxycarbonyl-1,4-phenylene-oxyterephthaloyl1,4-phenylcarbonyl), P7TOB, recorded at room temperature after rapidly cooling the sample from the mesophase. Scheme 1 shows the general structure for this series of polyesters prepared in our laboratory, where $\mathrm{X}=\mathrm{H}$ or $\mathrm{Cl}$, and $\mathrm{R}=\mathrm{a}$ linear or branched flexible spacer based on methylene, ethylene glycol or propylene glycol units. In P7TOB the spacer unit, $\mathrm{R}$ is $-\left(\mathrm{CH}_{2}\right)_{7}$.

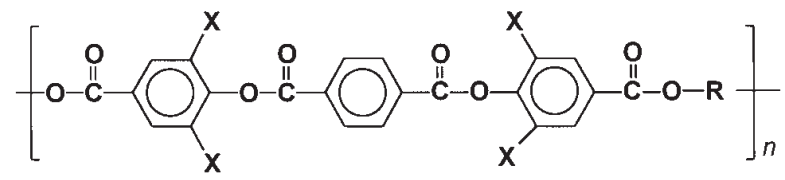

Scheme 1. General chemical structure

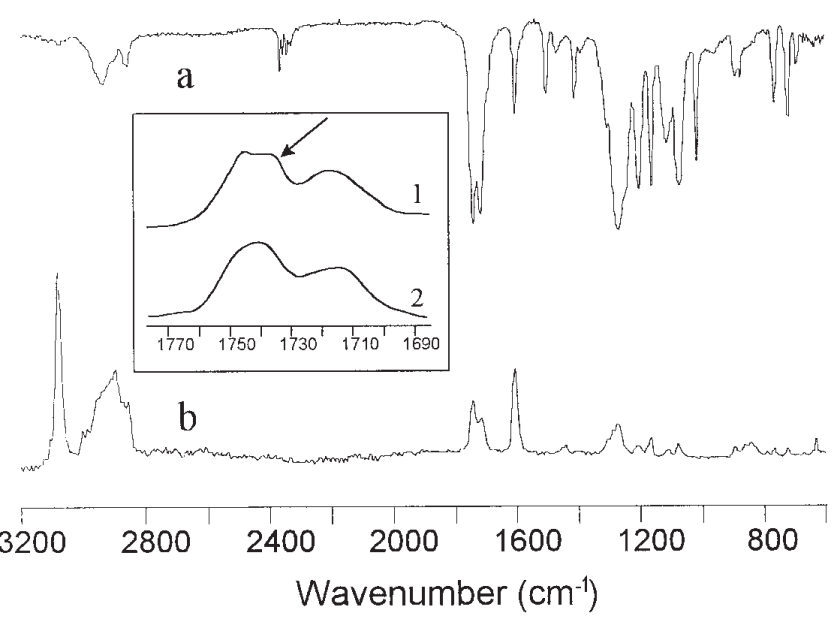

Figure 2. FT-IR (1: Aperture size $=25 \mu \mathrm{m} \times 25 \mu \mathrm{m})$ and FT-Raman (4) microspectra of the TLCP P7TOB, recorded at room temperature after rapidly cooling from the mesophase. Inset shows comparison of the Raman spectra of 1) the original, more crystalline sample, and 2) the quenched material. Arrow indicates the crystalline conformation of the terephthalate moeity.

As expected, the spectra of P7TOB are very similar to others in the series recorded using bulk sampling methods $[19,24,42]$, with characteristic bands appearing in both techniques due to both the mesogenic unit and the flexible spacer. The relative intensity of the carbonyl stretching modes of the terephthaloyl moiety of the mesogen at around $1740 \mathrm{~cm}^{-1}$, amongst others, has been shown to be associated with the distribution of crystalline and non-crystalline conformations, as shown in the inset. The increase in intensity of the lower frequency shoulder is characteristic of increasing crystallinity with, for example, annealing [19,42]. This type of crystalline conformation can be easily assigned in both techniques, and correlations can be clearly established between the DSC and X-ray data, and the vibrational spectroscopic evidence. In the case of the rapidly cooled sample considered in figure 2, the more symmetric profile of the terephthaloyl $\mathrm{C}=\mathrm{O}$ stretching vibration indicates that little crystalline material is encountered. In this sample, along with the disordered amorphous material, a "frozen-in" smectic mesophase is encountered, confirmed by WAXD $[19,40]$.

However, unlike the "crystalline" or "crystallinity bands" observed in many polymers, there are no specific bands related to a particular mesophase type that can provide a "liquid crystallinity" equivalent in TCLPs. This is unfortunate, since the nature and distribution of the liquid crystalline domains are important in the definition of the final properties of the material. However, vibrational microscopy is an ideal tool to study the conformational variations which occur during mesomorphic transitions, and in at least two cases $[19,22]$ variations in the conformational distribution of specific bands in the vibrational spectra of liquid crystalline phases have been related to a particular mesophase type, and 
provided evidence for its organisation. There are several testimonies in the literature describing the process of mesophase formation in main-chain TLCPs with aliphatic spacer groups of the above type. However, most authors seem to agree that the gradual inclusion of gauche conformations in the flexible spacer, such as the GTG" "kinks" proposed by Zerbi et al. [20], explain the majority of the changes observed, although concurrent variations in the conformation of the mesogenic unit cannot be ignored [21,24].

In order to illustrate several points, the thermotropic polyester poly(oxytetraethyleneoxide-oxycarbonyl-1,4-phenylene-oxyterephthaloyl-1,4-phenylcarbonyl), P4EGTOB, where the spacer unit, $\mathrm{R}$ (scheme 1) is $-\left(\mathrm{CH}_{2}-\mathrm{CH}_{2}-\mathrm{O}\right)_{4^{-}}$, is considered because of its highly complex transition behaviour $[43,56]$, as can be observed in the thermograms given in figure 2 in reference 43 for a series of heating and cooling cycles. This data combined with results from TOA and WAXD gives the approximate transitional diagram shown in scheme 2:

$$
\begin{aligned}
& 110^{\circ} \mathrm{C} \quad 160{ }^{\circ} \mathrm{C} \quad 210{ }^{\circ} \mathrm{C} \quad 240{ }^{\circ} \mathrm{C} \\
& \mathbf{K}_{\mathrm{I}} \text {-------- } \mathbf{K}_{\mathrm{II}}+\mathbf{S}_{\mathrm{E}} \text {-------- } \mathbf{S}_{\mathrm{A}} \text {-------- } \mathbf{N} \text {-------- } \mathbf{I}
\end{aligned}
$$

Scheme 2

The longer and more flexible spacer in this polymer seems to facilitate both two- and three-dimensional ordering when compared to others of the series. It should be noted that depending on the thermal history, both crystal forms and a smectic $S_{A}$ mesophase can be observed at room temperature [43]. The original sample is relatively crystalline, as can be observed from the FTIR and FT-Raman spectra at room temperature in figure 3, and crystallinity is easily recovered on cooling, even at very high cooling rates.

The spectra recorded by FT-IR microscopy as a function of temperature show significant variations over the whole temperature range (Fig. 4). Particularly, the dramatic increase in intensity at between $100-110{ }^{\circ} \mathrm{C}$ of the terephthaloyl carbonyl stretching mode at around $1731 \mathrm{~cm}^{-1}$, near the complicated transition zone observed in the DSC thermograms, which corresponds with the perfection of the more defective crystalline structure predominant at lower temperatures $\left(\mathrm{K}_{\mathrm{I}}\right)$, to a larger more perfect higher melting polymorph $\left(\mathrm{K}_{\mathrm{II}}\right)$. FT-IR microscopy shows this behaviour more acutely than FT-Raman due to the greater sensitivity to the environment of the $\mathrm{C}=\mathrm{O}$ stretch. Other bands observed in the micro-IR spectra which appear to be related with conformations in the crystalline material are observed at 1505 (sh), 1350,1306 (sh), 1213,888 , and $830 \mathrm{~cm}^{-1}$. At around $130{ }^{\circ} \mathrm{C}$, the $\mathrm{K}_{\mathrm{II}}$ form starts to melt, and a more disordered material results. The resulting spectral data manifests the disappearance of all the bands associated with the crystalline material. Further increase in temperature results in a series of bandshifts concomitant with the reduction in the magnitude and specificity of the interchain force constants [13]. The structural variations observed in the IR spectra at the smectic A to nematic $\left(\mathrm{S}_{\mathrm{A}}-\mathrm{N}\right)$ transition, at around $210^{\circ} \mathrm{C}$,

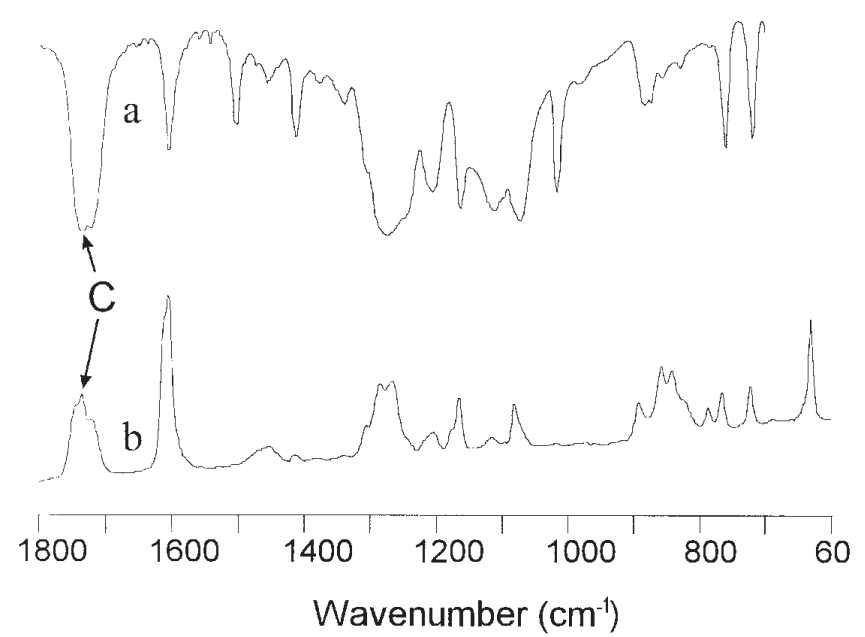

Figure 3. FT-IR (1: Aperture size $=50 \mu \mathrm{m} \times \mathbf{5 0} \mu \mathrm{m}$ ) and FT-Raman (2) spectra of the TLCP P4EGTOB, recorded at room temperature. $\mathrm{C}=$ crystalline conformation of terephthaloyl carbonyl stretch.

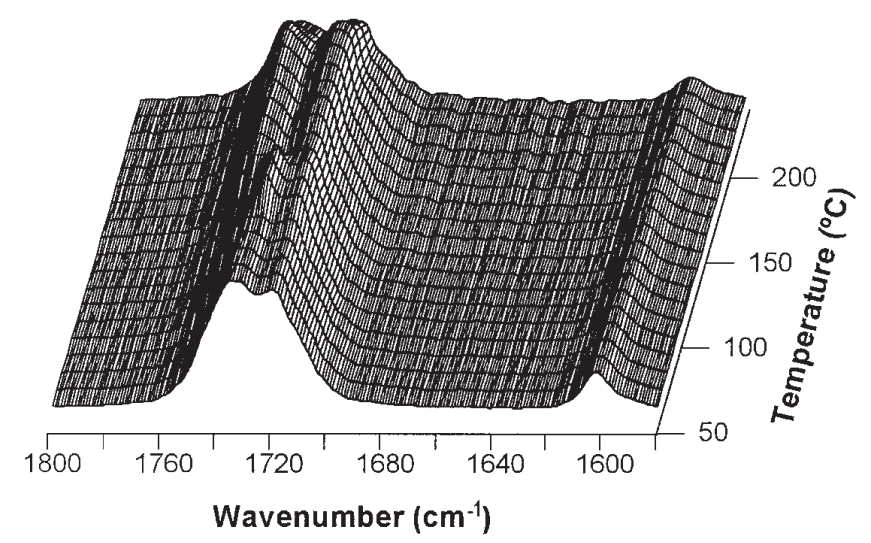

Figure 4. FT-IR microspectra of P4EGTOB in the range $1800-$ $1640 \mathrm{~cm}^{-1}$ as a function of temperature. 1: aperture size $=50 \mu \mathrm{m} \times 50 \mu \mathrm{m}$.

are subtle and difficult to characterise, but the absorbance of IR bands at various frequencies can be seen to vary dramatically at the $\mathrm{N}-\mathrm{I}$ phase transition. The thermal transitions can be clearly observed by following the absorbance of a variety of bands in the spectra, as shown in figure 5, and this data corresponds very well with the DSC and TOA data [43].

Time-resolved infrared microscopy (TRIM) permits the study of dynamic and isothermal kinetics of phase transformations which involve conformational differences, or variations in the intermolecular interactions of TLCP systems. Careful temperature control, ramping and rapid spectral acquisition is required, and reasonable results can be 
obtained with a variety of commercial cells and software. For more precise thermal control, the use of simultaneous FT-IR microscopy and microcalorimetry has been reported $[57,58]$, however, in some cases the kinetic study of the development of liquid crystalline phases or the disappearance of memory effects as a function of time are still possible with less sophisticated temperature cells due to the relatively long relaxation times observed in these materials.

\section{Orientational order}

Samples of oriented mesophases can be prepared by applying magnetic fields, or by mechanical processes such as extrusion accompanied by rapid cooling, but the control of the orientation is difficult and depends on a series of factors such as the preparation temperature, the molecular weight, the nature of the preparative surface, etc. There are several techniques available for the characterisation of orientational order. These include X-ray diffraction (widely used for TLCPs), birefringence, polarised fluorescence and polarised vibrational spectroscopy. Of the latter, IR dichroism is by far the most frequently used technique, and has been widely applied to determine bulk orientational order parameters in "combed" and drawn TCLPs [3,7]. and process oriented materials $[6,8,9,11]$. There are several FTIR microscopy studies of the degree of orientation in commercial systems [27-31], including the determination of orientation profiles and skin-core orientation differences. These approaches are common to the study of polymer orientation, are well documented, and will not be considered here.

\section{Polarised microscopy}

Using polarised visible light in the viewing mode of the vibrational microscope, it is possible to observe birefringent textures corresponding with the different types of liquid crystalline order, when sample preparation is optimised. Indeed TOA is the most commonly used method for the identification of liquid crystallinity and mesophase types. It is well known that when we apply polarised IR radiation to an anisotropic material, the intensities of the bands observed in the spectrum will be maximised when the polarisation direction is the same as that of the oscillating dipole moment of the given vibration. Thus, the combination of TOA with polarised vibrational microscopy presents important possibilities for the study of TLCPs, given that the microscopic domains corresponding with different levels of order can be easily isolated and characterised as a function of the polarisation conditions and the temperature or thermal and thermomechanical history of the material.

Considering as an example another polyester of the aforementioned series, with three propylene glycol units in the flexible spacer, PPG3TOB, a birefringent texture characteristic of a nematic mesophase, Williams domains, can be generated by cooling from the melt to around $180{ }^{\circ} \mathrm{C}$ at the biphasic transition region close to the clearing point [59].

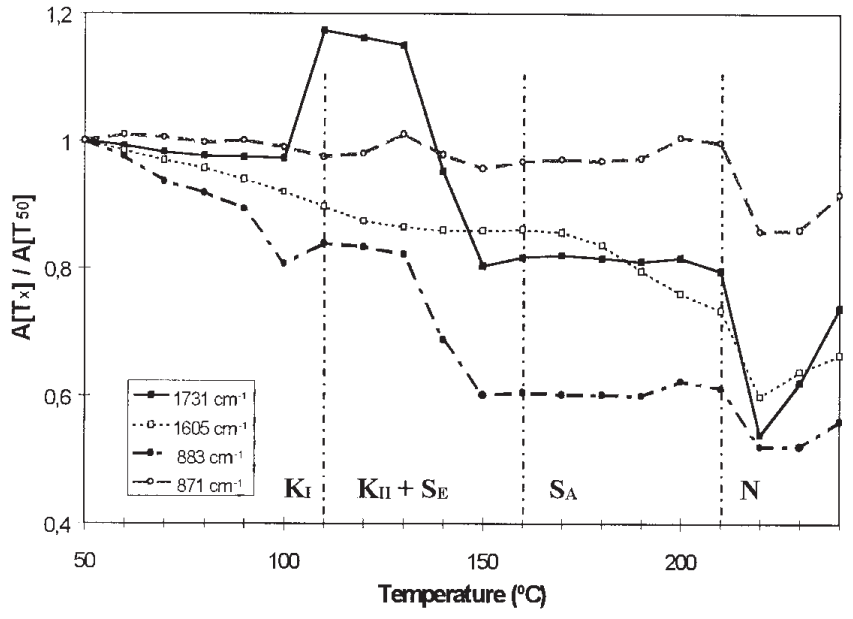

Figure 5. Absorbance ratios at selected frequencies in the FT-IR microspectra of P4EGTOB as a function of temperature, $T_{x^{*}}$ The transition temperatures [43] are indicated. $K_{1}$ and $\mathrm{K}_{\mathrm{II}}=$ polymorphs, $\mathrm{S}_{\mathrm{E}}+\mathrm{S}_{\mathrm{A}}=$ smectic mesophases, $\mathrm{N}=$ nematic mesophase. 1: aperture size $=50 \mu \mathrm{m} \times 50 \mu \mathrm{m}$.

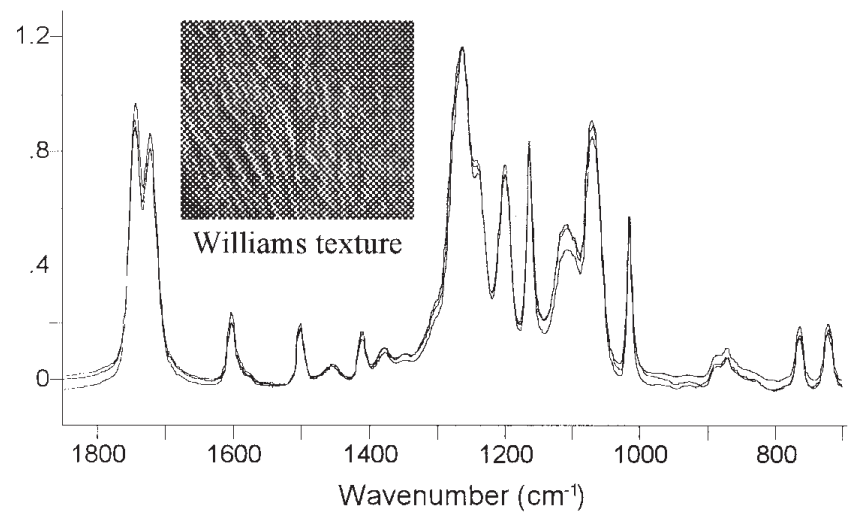

Figure 6. FT-IR microspectra of PPG3TOB recorded from different positions in the sample at $182^{\circ} \mathrm{C}$. 1: aperture size $=20 \mu \mathrm{m} \times 140 \mu \mathrm{m}$, recorded along the Williams texture bands, shown in the inset, and with the same aperture in the other zones.

This can be observed along with spectra recorded at this temperature in Figure 6. The texture band thickness in the microphotograph shown is around $20 \mu \mathrm{m}$. When recording the IR spectra from different locations in the sample, it was noted that no clear differences could be observed between the IR spectra of regions which manifested this recognisable birefringent texture, and regions at the same temperature where the texture was not observed (Fig. 6) and the only apparent differences arise from variations in the LCP film thickness. This is consistent with the fact that vibrational 
spectroscopy is sensitive to the conformational differences in the system which is a function of temperature. Liquid crystalline order is long range, although some evidence of specific interactions $[13,60]$ and conformations $[19,22]$ has been presented for some systems. However, using polarised IR radiation, it is possible to obtain more information on the anisotropic nature of the different domains. This is illustrated in figure 7 , where the normalised intensities of the $1605 \mathrm{~cm}^{-1}$ band, corresponding with a quadrant ring stretching mode characteristic of the long axis of the mesogenic unit, in the polarised spectra of PPG3TOB, is compared as a function of the polarisation angle for thermal histories. It can be clearly observed that in the isotropic state, there is no preferred orientational order of the mesogenic units, whereas at room temperature after rapidly cooling from the nematic phase some anisotropy is introduced into the material which consists of a small proportion of smectic mesophase along with crystalline and amorphous material [59]. However, in the polarised FT-IR microspectra recorded in the nematic mesophase a considerably higher degree of anisotropy can be observed, moreso after longer conditioning time in the mesophase.

To date, no unambiguous texture-structure correlations have been made for TLCPs. This is an important field not only for mesomorphic materials, but also for oriented polymer systems in general and process-orientated birefringent systems such as flowing polymer melts. Simple mapping techniques have already been used to study orientation profiles in TLCPs in the solid state [30]. By the combination of mapping techniques with variable angle polarisation it is possible to study anisotropic distribution in the sample. However, given that this type of analysis generates a very large amount of data at each sampling point, new processing methods or the application of sophisticated multivariate analysis to the resulting data will be necessary, and may provide some insight into the nature of the orientational order in these materials, and generate a model for future advances in the understanding of ordered and disordered states in condensed phases.

\section{Final comment}

Over the next years, it is expected that the advances in vibrational microscopy instrumentation and methodology will provide new solutions to the study of thermotropic behaviour in TLCPs, and provide us with evidence for a greater understanding of the special nature of these materials.

\section{Acknowledgements}

We wish to thank both Dr. T. Jawhari-Colin (SCT, Universitat de Barcelona, Spain) and Dr. S. Sánchez Cortés (Instituto de Estructura de la Materia, CSIC, Madrid, Spain) for the spectra obtained on their Raman microscope systems.
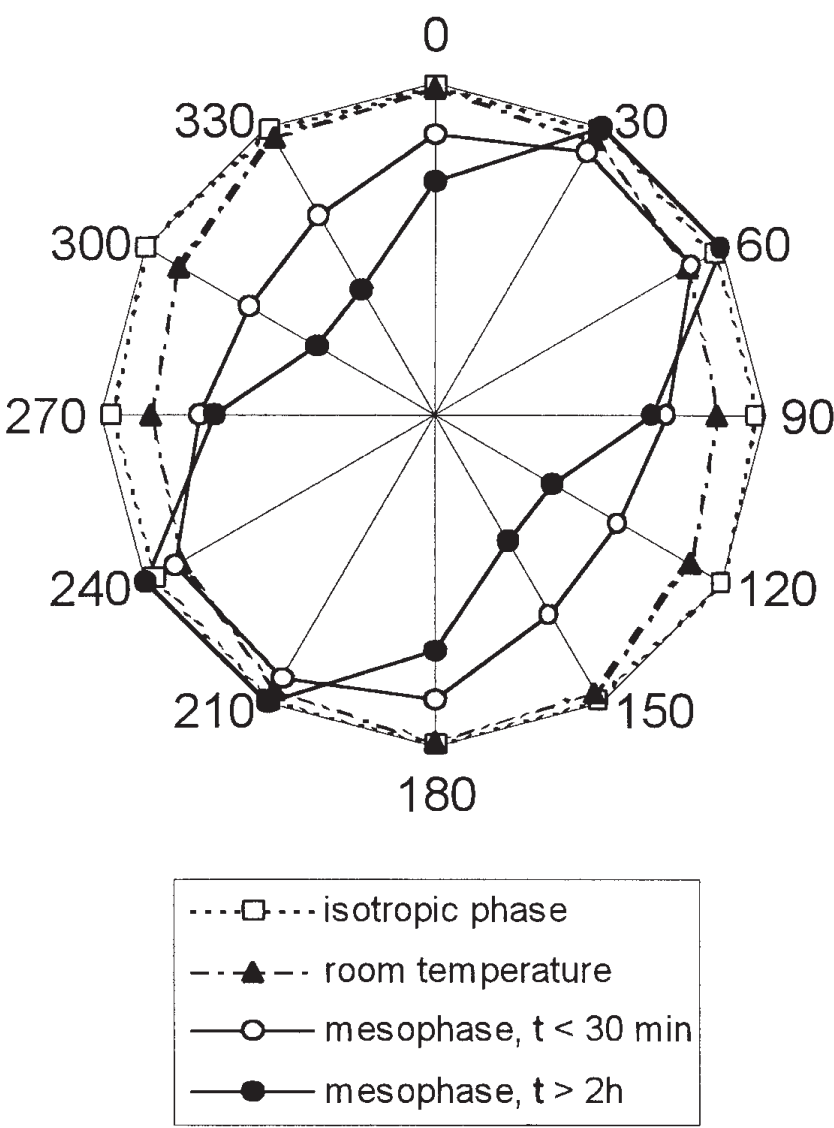

Figure 7. Radial graph of normalised absolute absorbance of the $1605 \mathrm{~cm}^{-1}$ ring mode from the FT-IR microspectra of PPG3TOB, versus polarisation angle for different thermal histories. 1: aperture size $=\mathbf{5 0} \mu \mathrm{m} \times \mathbf{5 0} \mu \mathrm{m}$.

\section{References}

1. Blumstein, A. Polymeric Liquid Crystals; Plenum: New York, 1985.

2. Marco, C.; Lorente, J.; Gómez, M.A.; Fatou, J.G. Polymer 1992, 33, 3108 .

3. Volchek, B.Z.; Kholmuradov, N.S.; Purkina, A.V.; Bilibin, A.Yu.; Skorokhodov, S.S. Polym. Sci. USSR 1985, 27, 90.

4. Volchek, B.Z.; Kholmuradov, N.S.; Purkina, A.V.; Bilibin, A.Yu.; Skorokhodov, S.S. Polym. Sci. USSR 1986, 28, 1674.

5. Hu, S.; Xu, M.; Wang, X.; Lenz, R.W.; Stein, R.S. Polymer 1988, 29, 789 .

6. Pirnia, A.; Sung, C.S.P. Macromol. 1988, 21, 2699.

7. Volchek, B.Z.; Medvedeva, D.A.; Purkina, A.V.; Shilov, S.V. Polym. Sci. USSR 1990, 32, 1462.

8. Kaito, A.; Nakayama, K. Macromol. 1992, 25, 4882.

9. Kaito, A.; Kyotani, M.; Nakayama, K. J. Appl. Polym. Sci. 1995, 55, 1489.

10. Voyiatzis, G.; Petekidis, G.; Vlassopoulos, D.; Kamitsos, E.I.; Bruggeman, A. Macromol. 1996, 29, 2244. 
11. Wiberg, G.; Gedde, U.W. Polymer 1997, 38, 3753.

12. Galli,G.; Benedetti, E.; Chiellini, E.; Ober, C.; Lenz, R.W. Polym. Bull. 1981, 5, 497.

13. Wu, P.P.; Hsu, S.L.; Thomas, O.; Blumstein, A. J. Polym. Sci. Part B: Polym. Phys. 1986 24, 827.

14. Abdullin, S.N.; Furer, V.L.; Bilibin, A.Y. Piraner, O.N. J. Appl. Spectrosc. 1989, 50, 423.

15. Benedetti, E.; Galleschi, F.; Chiellini, E.; Galli, G.; Lenz, R.W. J. Polym. Sci. Polym. Phys. Ed. 1989 27, 25.

16. Jedlinski, Z.; Franek, J.; Kulczycki, A.; Sirigu, A.; Carfagna, C. Macromol. 1989, 22, 1600.

17. Abdullin, S.N.; Furer, V.L.; Bilibin Polym. Sci. Tech. 1991, 18(3), 89.

18. Galbiati, E.; Zerbi, G.; Benedetti, E.; Chiellini, E. Polymer 1991, 32, 1555

19. Ellis, G.; Lorente, J.; Marco, C.; Gómez, M.A.; Fatou, J.G.; Hendra, P.J. Spectrochim. Acta 1991, 47A, 1353.

20. Ellis, G.; Gómez, M.A.; Marco, C.; Fatou, J.G. Polym. Bull. 1991, 25, 351.

21. Shilov, S.V.; 18. Volchek, B.Z.; Zuev, V.V.; Skorokhodov, S.S. Macromol. Chem. Phys. 1994, 195, 865.

22. Silvestri, R.L.; Koenig, J.L. Polymer 1994, 35, 2528.

23. Moriya, K.; Minagawa, T.; Yano, S. Polym. Bull. 1994, 33, 209.

24. Ellis, G.; Marco, C.; Del Pino, J.; Gómez, M.A.; Fatou, J.G.; Vib. Spectrosc. 1995, 9, 49.

25. Campoy, I. Spectrochim. Acta 1996, 52A, 619.

26. Campoy, I. Vib. Spectrosc. 1996, 13, 99.

27. Kaito, A.; Kyotani, M.; Nakayama, K. Macromol. 1991, 24, 3244.

28. Kaito, A.; Kyotani, M.; Nakayama, K. Polymer 1992, 33, 2672.

29. Kaito, A.; Kyotani, M.; Nakayama, K. J. Appl. Polym. Sci. 1993, 48, 2147.

30. Jansen, J.A.J.; Paridaans, F.N.; Heynderickx, I.E.J. Polymer 1994, 35, 2970

31. Kawagoe, M.; Nomiya, M; Qiu, J.; Morita, M.; Mizuno, W. Polymer 1997, 38, 113.

32. Messerschmidt, R.G.; Harthcock, M.A. (Eds.) Infrared Microscopy. Theory and Applications; Marcel Dekker: New York, 1988.

33. Pastor, J.M. Macromol. Chem., Macromol. Symp. 1991, 52, 57.

34. Katon, J.E. Vib. Spectrosc. 1994, 7, 201.

35. Chalmers, J.M.; Everall, N.J. Macromol. Symp. 1995, 94, 33.

36. Buffeteau, T.; Desbat, B.; Eyquem, D. Vib. Spectrosc. 1996, $11,29$.
37. Markwort, L.; Kip, B. J. Appl. Polym. Sci. 1996, 61, 231.

38. Chalmers, J.M.; Everall, N.J.; Hewitson, K.; Chesters, M.A.; Pearson, M.; Grady, A.; Ruzicka, B. Analyst 1998, 123, 579.

39. Griffin A.C; Johnson, J.E. (Eds.) Liquid Crystals and Ordered Fluids, Plenum: New York, 1984, Vol. 4.

40. Marco, C.; Lorente, J.; Gómez, M.A.; Fatou, J.G. Polymer 1992, 33, 3108.

41. Del Pino, J.; Marco, C.; Gómez, M.A.; Fatou, J.G. Makromol. Chem. 1992, 193, 2251.

42. Del Pino, J.;Gómez, M.A.; Marco, C.; Ellis, G.; Fatou, J.G. Macromol. 1992, 25, 4642.

43. Gómez, M.A.; Marco, C.; Fatou, J.G.; Suárez, N.; Laredo, E.; Bello, A. J. Polym. Sci: Part B: Polym. Phys. Ed. 1995, 33, 1259.

44. Bañares, M. Personal communication.

45. Cutler, D.J.; Petty, C.J. Spectrochim. Acta 1991, 47A, 1159.

46. Bennett, R. Spectrochim. Acta 1994, 50A, 1813.

47. Cooper, J.B.; Wise, K.L.; Jensen, B.J. Anal. Chem. 1997, 69 , 1973.

48. Harthcock, M.A.; Atkin, S.C.; Davis, B.L. Microbeam Anal. 1988, 23, 203.

49. Garton, A.; Batchelder, D.N.; Cheng, C. Appl. Spectrosc. 1993, 47, 922.

50. Kressler, J; Schäfer, R.; Thomann, R. Appl. Spectrosc. 1998, $52,1269$.

51. Ward, K.J. in Microbeam Analysis, Proc. 28th Annual MAS Meeting, New Orleans, 1994, 79.

52. Webster, S.; Batchelder, D.N.; Smith, D.A. Appl. Phys. Lett. 1998, 72, 1478.

53. Webster, S.; Smith, D.A.; Batchelder, D.N. Vibr. Spectrosc. 1998, 18, 51.

54. Piednoir, A.; Licoppe, C.; Creuzet, F. Opt. Commun. 1996, $129,414$.

55. Lahrech, A.; Bachelot, R.; Gleyzes, P.; Bocarra, A.C. Opt. Lett. 1996, 21, 1315 .

56. Galli, G.; Chiellini, E.; Torquati, G.; Caciuffo, R.; Melone, S.; Gallot, B. Polym. J. 1989, 21, 155.

57. Mirabella, F.M. Pract. Spectrosc. 1988, 6, 85.

58. Mirabella, F.M., Jr.; Shankernarayaran, M.J. Polym. Mater. Sci. Eng. 1988, 59, 450.

59. Marco Rocha, C.; Gómez, M.A.; Fatou, J.G.; Evans, P.; Cronauer, J.; Zachmann, G. Polymer 1997, 38, 1601.

60. Galbiati, E.; Del Zoppo, M.; Tieghi, G.; Zerbi, G. J. Chem. Phys. 1993, 34, 1807. 